\title{
Aditivos presentes en etiquetado de los Nuggets de pollo
}

\author{
Additives present in the label of Chicken Nuggets
}

\author{
Michel E. Flores-Chávez ${ }^{a}$, Italletzy Mejía-Hernández ${ }^{b}$, Alicia Cervantes-Elizarrarás ${ }^{c}$, \\ Aranza Y. Pimentel-Munguía ${ }^{d}$ y Esther Ramírez-Moreno ${ }^{e}$
}

\begin{abstract}
:
Chicken Nuggets have almost half of breaded (47\%), so it is detrimental to the amount of protein. The amount of fat and fat is high because they are used in the breading of the product, so its price is high compared to fresh poultry products. Therefore, the consumption of these products should be limited, especially because of their high sodium content and additives. The present work evaluated the nutritional composition and listed the additives present in the labeling in Chicken Nuggets. A visit was made to various supermarkets (Aurrera, Soriana, Wal-Mart), to evaluate nutritional information, additive content and price considering 4 brands of Chicken Breast Nuggets. In order to evaluate the nutritional composition and list the additives present in the labeling in Chicken Nuggets, a portion of fresh chicken breast was used to make a comparison between the nutritional composition of the Chicken Nuggets. The\% of meat present in chicken Nuggets was obtained by removing and weighing the breaded and subtracting from the total unit
\end{abstract}

\section{Keywords:}

Food additive, dextrose, leptin.

\section{Resumen:}

Los Nuggets de pollo presentan casi la mitad de empanizado (47\%), por lo presenta un detrimento de la cantidad de proteínas. La cantidad de grasa y grasa es alta debido a que se utilizan en el empanizado del producto, por lo cual su precio es alto en comparación con los productos avícolas frescos. Por lo tanto, el consumo de estos productos debe ser limitado, sobre todo por su alto contenido de sodio y aditivos. El presente trabajo evaluó la composición nutrimental y enlistó los aditivos presentes en el etiquetado en Nuggets de pollo. Se realizó una visita a diversos supermercados (Aurrera, Soriana, Wal-Mart), para evaluar la información nutrimental, contenido de aditivos y precio considerando 4 marcas de Nuggets de Pechuga de Pollo. Con el objetivo de evaluar la composición nutrimental y enlistar los aditivos presentes en el etiquetado en Nuggets de pollo. Para establecer una comparación entre la composición nutrimental de los Nuggets de pollo se utilizó una porción de pechuga de pollo fresco. El \% de carne presente en los Nuggets de pollo se obtuvo retirando y pesando el empanizado y restando de la unidad total.

\section{Palabras Clave:}

Aditivo alimentario, dextrosa, leptina.

\footnotetext{
a Autor de correspondencia, Universidad Autónoma del Estado de Hidalgo, Instituto de Ciencias de la Salud, https://orcid.org/0000-0003-0525-2293, Email: ekaterine970223@gmail.com

${ }^{\mathrm{b}}$ Universidad Autónoma del Estado de Hidalgo, Instituto de Ciencias de la Salud, https://orcid.org/0000-0002-4721-1644, Email: italletzy.mh.96@gmail.com

c Universidad Autónoma del Estado de Hidalgo, Instituto de Ciencias de la Salud, http://orcid.org/0000-0002-1432-2882, Email: alicia_cervantes@uaeh.edu.mx

${ }^{\mathrm{d}}$ Universidad Autónoma del Estado de Hidalgo, Instituto de Ciencias de la Salud, https://orcid.org/0000-0002-1999-1540, Email: aranzapimentel1@gmail.com

${ }^{e}$ Universidad Autónoma del Estado de Hidalgo, Instituto de Ciencias de la Salud, http://orcid.org/0000-0002-9928-8600,

Email: rme1234@yahoo.com
} 


\section{Introducción}

La NMX-FF-080-SCFI-2006 establece que el pollo fresco es el que ha sido procesado y mantenido a una temperatura entre $0^{\circ} \mathrm{C}$ a $4^{\circ} \mathrm{C}$.

De acuerdo a la NOM-213-SSA1-2000, los productos cárnicos empanados o rebozados congelados son elaborados con carne molida, picada o en piezas, con adición o no de tejido graso, subproductos y aditivos, que pueden recibir un tratamiento térmico durante su elaboración, pero que necesitan ser cocinados para consumirlos, entre los que se encuentran los Nuggets de pollo.

Los Nuggets de pollo fueron introducidos en el mercado como piezas sólidas de carne de pechuga, que se troceaban en forma de triángulo y, tras el empanizado, eran sometidas a fritura. Sin embargo, este proceso original ha derivado actualmente en otras formas de elaboración. La creciente demanda ha provocado una necesidad continua de emplear nuevas materias primas y usar tecnologías que permitan mejorar su aspecto y también sus rendimientos. Como resultado, la cantidad de pollo en ellos ha disminuido y no se compone necesariamente de la pechuga: algunas marcas también suelen utilizar pierna, muslo, carne deshuesada de otras partes del pollo, pasta de pollo e incluso, piel. Además, se agregan otros ingredientes 0 aditivos como agua, aceite vegetal, harinas, almidones, sal, saborizantes, especias y proteína de soya (Bonato, P., Perlo, F., Teira, G., Soraya, R.S., 2006).

De acuerdo a la NOM-213-SSA1-2018 un aditivo alimentario es cualquier sustancia que no se consume normalmente como alimento, ni tampoco se usa como ingrediente básico en alimentos, tenga 0 no valor nutritivo, y cuya adición al producto con fines tecnológicos en sus fases de producción, elaboración, preparación, tratamiento, envasado, empaquetado, transporte o almacenamiento. Se agrega intencionalmente a los alimentos y bebidas en cantidades mínimas con objetivo de modificar sus caracteres organolépticos o facilitar o mejorar su proceso de elaboración o conservación.

\section{Objetivo}

Evaluar la composición nutrimental y enlistar los aditivos presentes en el etiquetado en Nuggets de pollo.

\section{Metodología}

Se realizó una visita a diversos supermercados (Aurrera, Soriana, Wal-Mart), para evaluar la información nutrimental, contenido de aditivos y precio considerando 4 marcas de Nuggets de Pechuga de Pollo. Para establecer una comparación entre la composición nutrimental de los Nuggets de pollo se utilizó una porción de pechuga de pollo fresco. El \% de carne presente en los Nuggets de pollo se obtuvo retirando y pesando el empanizado y restando de la unidad total.

\section{Resultados y discusión}

Los Nuggets se caracterizan por tener una capa de empanizado compuesta principalmente por harinas, grasas y huevo. El empanizado puede representar poco más del $50 \%$ del producto afectando la calidad nutricional del producto. En la tabla 1 se muestran los resultados de la prueba de \% de carne, en la cual se observó que el producto de la marca Tyson tiene el porcentaje más alto de contenido cárnico $(47.77 \%)$, mientras que Pilgrims presentó el más bajo en comparación con las otras marcas. De esta manera se denotó el alto porcentaje de empanizado que contienen estos productos.

El contenido proteico de los productos cárnicos debe oscilar alrededor del 22\% (FAO, 2005), lo cual se puede observar en la pechuga de pollo con un contenido del $19.75 \%$. En el caso de los Nuggets el porcentaje de proteína fue de $12-13 \%$, ya que depende de la presencia de empanizado, piel, soya, etc.

La pechuga de pollo contiene $1.5 \%$ de grasa mientras que los Nuggets presentaron de $14-21 \%$ de este componente, a excepción del producto Bachoco que presentó un $6 \%$, posiblemente debido a su gran contenido de soya.

En cuanto a $\mathrm{HCO}$, la pechuga de pollo no presenta cantidad alguna mientras que los Nuggets de Bachoco presentan la cantidad más elevada (21.19) y Great Value con la más baja (13 gr), la presencia de este macronutriente se atribuye al contenido de harinas, dextrosa, soya y almidón en el caso de Tyson.

Respecto al costo, los Nuggets de Great Value son los más económicos (\$9.8/100 gr), Tyson y Bachoco los más caros (\$12.7/100 gr); $y$ todos son de costo superior al de la Pechuga de pollo fresco $(\$ 7.10 / 100 \mathrm{gr})$.

Estos productos son altos en sodio ya que presentan cantidades por encima de las recomendadas para los productos bajos en sodio (>140 mg/porción). (NOM-086SSA1-1994))

Tabla 1. Información nutrimental de Pechuga de pollo fresca y Nuggets de pollo por $100 \mathrm{~g}$.

\begin{tabular}{lcccccccc}
\hline & $\begin{array}{c}\text { \% } \\
\text { carne }\end{array}$ & Kcal & Proteina & $\begin{array}{c}\text { Grasal } \\
\text { saturada }\end{array}$ & HCO & Fibra & $\begin{array}{c}\mathrm{Na} \\
\mathrm{mg}\end{array}$ & $\begin{array}{c}\text { Precio } \\
\$\end{array}$ \\
\hline Promedio & 100 & 97.5 & 19.75 & $1.5 / 0$ & 0 & 0 & 57.75 & 7.4 \\
\hline Great & & \multicolumn{7}{c}{ Nuggets de pollo } \\
Value & 44.9 & 230 & 13 & $14 / 5$ & 13 & 1 & 820 & 9.85 \\
Bachoco & 45.6 & 194 & 12.07 & $6.82 / 1.1$ & 21.19 & 5.64 & 620.6 & 12.70 \\
Tyson & 47.7 & 260 & 12 & $16 / 9$ & 17 & 1 & 588 & 12.77 \\
Pilgrims & 40.7 & 309 & 13.10 & $21.27 / 5.1$ & 16.17 & 0.30 & 548.2 & 11.28 \\
\hline
\end{tabular}

Como se puede observar en la tabla 2, los Nuggets de la marca Great Value y Bachoco, presentaron como ingrediente principal pechuga de pollo, pero también 
presentaron otros ingredientes como en la marca Tyson carne de la costilla y en el producto Pilgrims piel de pollo extra a la pechuga.

La NOM-213-SSA1-2002, regula los aditivos permitidos para la industrialización de estos productos. De acuerdo a la tabla 2, la marca Tyson presentó el mayor número de aditivos (12) a diferencia de Pilgrims que únicamente contiene 6 . Cabe resaltar que ninguna marca indica en su empaque la concentración de los aditivos.

Las 4 marcas de Nuggets ocupan dextrosa como aditivo, el cual es utilizado para inhibir el crecimiento microbiano mediante la reducción de la aw y el aumento de la presión osmótica (Badui, 2006). Este aditivo permite una mayor vida útil en conjunto con la congelación y refrigeración. Este parece ser parte del método de conservación

El glutamato monosódico está presente en la mayoría de las marcas potencializando la palatabilidad, en este caso intensifica los sabores umami (Badui, 2006) de los Nuggets de pollo, haciéndolos altamente apetecibles.

Otros aditivos encontrados en los Nuggets son los fosfatos de sodio (tripolifosfato de sodio), aluminio y calcio, utilizados como estabilizadores en la retención de líquidos de los alimentos y la extracción de proteínas miofibrilares produciendo la ligazón intermuscular (Schmidt,1984), lo que les brinda un aspecto muy agradable con bordes definidos, de un volumen aceptable y podría ser la razón por la que a pesar de no ser $100 \%$ carne de pollo tengan una buena adherencia el resto de sus ingredientes.

La OMS y el Codex Alimentarius han realizado estudios toxicológicos para determinar las cantidades permitidas en el uso de aditivos realizando recomendaciones para su consumo. Sin embargo, el consumo de aditivos en cantidades excesivas de manera frecuente se relaciona con afecciones a la salud, por ejemplo la Terbutilhidroquinona y el óxido de titanio presentes en los Nuggets de Great Value, están prohibidos en algunos países, debido a que generan daños cromosómicos desencadenando cáncer y problemas del sistema inmunológico (Chirino, L., 2018; Mejía, D-P., Pérez, M. \& Rosas, M., 2014). El glutamato monosódico interfiere con la producción de leptina creando una adicción que nos impulsa a seguir comiendo (Aza, J. \& Restrepo L., 2012), y se asocia con el síndrome del restaurante Chino, caracterizado por palpitaciones, dolores musculares y de cabeza después de un consumo excesivo e incluso puede ser un desencadenante de gota ya que durante su metabolismo se produce ácido úrico (Badui, 2006).
Tabla. 2 Ingredientes y aditivos de Nuggets de pechuga de pollo

\begin{tabular}{|c|c|c|c|c|}
\hline \multicolumn{5}{|c|}{ MARCAS } \\
\hline & Great Value & Barroco & Pilgrims & Tyson \\
\hline \multicolumn{5}{|c|}{ INGREDIENTES } \\
\hline Huevo & $x$ & $\mathrm{x}$ & $\mathrm{x}$ & $\mathrm{x}$ \\
\hline Soya & $\mathrm{x}$ & $\mathrm{x}$ & & \\
\hline Pechuga de pollo & $\mathrm{x}$ & $\mathrm{x}$ & $\mathrm{x}$ & $\mathrm{x}$ \\
\hline Piel de pollo & $\mathrm{x}$ & & $\mathrm{x}$ & \\
\hline Carne de costilla & & & & $\mathrm{x}$ \\
\hline Levadura & $\mathrm{x}$ & $\mathrm{x}$ & $\mathrm{x}$ & \\
\hline Sal Yodada & $\mathrm{x}$ & $\mathrm{x}$ & $\mathrm{x}$ & \\
\hline Azúcar & $\mathrm{x}$ & $\mathrm{x}$ & $\mathrm{x}$ & \\
\hline Harina de maíz & $\mathrm{x}$ & $\mathrm{x}$ & $\mathrm{x}$ & \\
\hline Harina de arroz & & & & $\mathrm{x}$ \\
\hline Harina de Trigo & $\mathrm{x}$ & $\mathrm{x}$ & & $\mathrm{x}$ \\
\hline \multicolumn{5}{|c|}{ ADITIVOS } \\
\hline Dextrosa & $\mathrm{x}$ & $x$ & $\mathrm{x}$ & $\mathrm{x}$ \\
\hline Paprika & $\mathrm{x}$ & $\mathrm{x}$ & & $\mathrm{x}$ \\
\hline Cúrcuma & & & & $x$ \\
\hline Ajo & & & & $\mathrm{x}$ \\
\hline Almidón & & & & $\mathrm{x}$ \\
\hline Goma Guar & $\mathrm{x}$ & & & \\
\hline Saborizante natural & $\mathrm{x}$ & & & $\mathrm{x}$ \\
\hline Saborizante artificial & $\mathrm{x}$ & $\mathrm{x}$ & $\mathrm{x}$ & \\
\hline Tripolifosfato de sodio & $\mathrm{x}$ & $\mathrm{x}$ & $x$ & $\mathrm{x}$ \\
\hline Óxido de titanio & $\mathrm{x}$ & & & \\
\hline Ternuthihidroquinona & $\mathrm{x}$ & & & \\
\hline Glutamato monosódico & $\mathrm{x}$ & $\mathrm{x}$ & $\mathrm{x}$ & \\
\hline Fosfato de sodio & & $\mathrm{x}$ & & \\
\hline Carrangenina & & $\mathrm{x}$ & & \\
\hline Annato & & $\mathrm{x}$ & & \\
\hline Bicarbonato de sodio & & & $\mathrm{x}$ & $\mathrm{x}$ \\
\hline Silicio & & & $\mathrm{x}$ & \\
\hline Fosfato de aluminio & & & & $\mathrm{x}$ \\
\hline Fosfato monocálcico & & & & $\mathrm{x}$ \\
\hline
\end{tabular}

\section{Conclusión}

Los Nuggets de pollo presentan casi la mitad de empanizado (47\%), por lo presenta un detrimento de la cantidad de proteínas. La cantidad de grasa y grasa es alta debido a que se utilizan en el empanizado del producto, por lo cual su precio es alto en comparación con los productos avícolas frescos. Por lo tanto, el consumo de estos productos debe ser limitado, sobre todo por su alto contenido de sodio y aditivos. 


\section{Referencias}

1. Aza, J. \& Restrepo L. (Enero-Abril, 2012) El glutamato monosódico: influencia de su consumo sobre algunos factores metabólicos de ratones y en el aumento de la apetencia Vitae, Universidad de Antioquia Medellín, Colombia; 19(1), S294S296

2. Badui, S. (2006). Química de los alimentos. México: Pearson educación de México, S.A. de C. V.

3. Bonato, P., Perlo, F., Teira, G., Soraya, R.S. 2006. Características texturales de Nuggets de pollo elaborados con carne de ave mecánicamente recuperada en reemplazo de carne manualmente deshuesada. Rev. Cien Doc. y Tecno. \#32: 219239 PP.

4. Chirino, L. (2018). Efectos negativos del dióxido de titanio en la salud humana. Gaceta UNAM. Núm. 5076. Recuperado de: http://www.gaceta.unam.mx/efectos-negativos-del-dioxido-detitanio-en-la-salud-humana/

5. FAO (2005). Departamento de Agricultura y Sanidad Animal. Composición de la carne. Recuperado de: http://www.fao.org/ag/againfo/themes/es/meat/backgr_composi tion.html

6. Mejía, D-P., Pérez, M. \& Rosas, M. (2014). ¡Alerta! Tbhq en alimentos con grasa. La Ciencia y el Hombre, 27(3), 34-38.

7. NMX-FF-080-SCFI-2006. Productos avícolas - carne de pollo de engorda en canal y en piezas - clasificación)

8. NOM-086-SSA1-1994. Alimentos y bebidas no alcohólicas con modificaciones en su composición. Especificaciones nutrimentales

9. NOM-213-SSA1-2000. Productos y servicios. Productos cárnicos procesados. Diario Oficial de la Federación. México, D.F

10. NOM-213-SSA1-2018. Diario Oficial de la Federación. México, D.F

11. Schmidt, H. (1984). Carne y productos cárnicos su tecnología y análisis. Santiago de Chile: Editorial Universitaria. 